\title{
In vitro fluorescence studies of transcription factor IIB-DNA interaction*
}

\author{
Andrzej Górecki ${ }^{\bowtie}$, Małgorzata Figiel and Marta Dziedzicka-Wasylewska \\ Department of Physical Biochemistry, Faculty of Biochemistry, Biophysics and Biotechnology, Jagiellonian University, Kraków, Poland
}

\begin{abstract}
General transcription factor TFIIB is one of the basal constituents of the preinitiation complex of eukaryotic RNA polymerase II, acting as a bridge between the preinitiation complex and the polymerase, and binding promoter DNA in an asymmetric manner, thereby defining the direction of the transcription. Methods of fluorescence spectroscopy together with circular dichroism spectroscopy were used to observe conformational changes in the structure of recombinant human TFIIB after binding to specific DNA sequence. To facilitate the exploration of the structural changes, several site-directed mutations have been introduced altering the fluorescence properties of the protein. Our observations showed that binding of specific DNA sequences changed the protein structure and dynamics, and TFIIB may exist in two conformational states, which can be described by a different microenvironment of W52. Fluorescence studies using both intrinsic and exogenous fluorophores showed that these changes significantly depended on the recognition sequence and concerned various regions of the protein, including those interacting with other transcription factors and RNA polymerase II. DNA binding can cause rearrangements in regions of proteins interacting with the polymerase in a manner dependent on the recognized sequences, and therefore, influence the gene expression.
\end{abstract}

Key words: transcription factors, TF2B, TFIIB, fluorescence spectroscopy, fluorescence quenching, site directed mutagenesis

Received: 14 March, 2015; revised: 20 April, 2015; accepted: 20 May, 2015; available on-line: 18 August, 2015

\section{INTRODUCTION}

General transcription factor IIB (TFIIB) is one of the basal constituents of the preinitiation complex of eukaryotic RNA polymerase II, which synthesizes mRNAs and snRNAs. The role of the TFIIB protein is recognition of specific DNA sequences within the promoter region as well as recruitment and proper positioning of RNA polymerase II (RNAPII). As a monomeric protein requiring no post-translational modifications, TFIIB can be expressed in a prokaryotic system in a fully functional form (Deng \& Roberts, 2007). TFIIB molecule comprises two domains of different functions, yet affecting each other. The N-terminal domain (NTD) contains a $\beta$-sheet and a $\mathrm{C}_{3} \mathrm{H}$ type zinc finger (Chen et al., 2000) that binds to RNAPII near the active site (Kostrewa et al., 2009). The C-terminal domain (CTD) of TFIIB interacts with promoter DNA, TATA-box binding protein (TBP) and numerous transcription activators (Deng \& Roberts, 2007). The two DNA-binding motifs are imperfect direct repeats of cyclin-like structure (Bagby et al.,
1995; Malik et al., 1993), that specifically recognize two promoter fragments, located immediately upstream and downstream of the TATA box (Lagrange et al., 1998; Tsai \& Sigler, 2000). The binding is direct (Lagrange et al., 1998) but simultaneous interaction at both sites is enabled only by previous binding of TBP that bends the DNA molecule about 80 (Lee \& Hahn, 1995; Nikolov et al., 1995).

TFIIB molecule can exist in several conformations. Firstly, the orientation of the two cyclin-like repeats is different for the free CTD NMR structure (Bagby et al., 1995) and the X-ray structure of the CTD complexed with TBP and DNA (Nikolov et al., 1995). Moreover, the whole protein can adopt either closed or open form, due to an intra-molecular binding between NTD and CTD (Grossmann et al., 2001; Hayashi et al., 1998; Roberts \& Green, 1994). The equilibrium between those conformations regulates TFIIB function within the preinitiation complex (Hawkes et al., 2000). The closed conformation has been shown to stabilize TFIIB binding to TBP and DNA in vitro (Bangur et al., 1999; Zhang et al., 2000), while the open conformation promotes transcriptional activation (Hawkes et al., 2000) and is stabilized by a number of transcription activators like acidic activator (Roberts \& Green, 1994), VP16 10712906, Vpr (Agostini et al., 1999), and Pho4 (Wu \& Hampsey, 1999).

Although crucial for proper course of transcription, the TFIIB-DNA interaction is not yet thoroughly understood. Therefore we aimed to investigate what structure changes in TFIIB accompany DNA binding. Two sequences: adenovirus major late promoter (AdML) and adenovirus E4 promoter (AdE4), were chosen as examples for interaction studies. They differ in their agreement with the consensus sequence (Deng \& Roberts, 2005; Lagrange et al., 1998), with AdML being closer to the consensus. Both sequences are commonly used for investigating transcription initiation in Eucaryota (Deng

e-mail: andrzej.gorecki@uj.edu.pl

* Preliminary report on the same subject has been presented during the 42nd Winter School of Faculty of Biochemistry, Biophysics and Biotechnology, Zakopane 10-14 February 2015

Abbreviations: AdE4, adenovirus E4 promoter; AdML, adenovirus major late promoter; ANOVA, analysis of variance; BREu, IIB recognition element upstream of TATA; BREd, IIB recognition element downstream of TATA; BSA, bovine serum albumin; CRP, prokaryotic transcription factor cyclic AMP Receptor Protein from E.coli; CTD, C-terminal domain; DMSO, dimethyl sulfoxide; DTT, dithiothreitol; IPTG, isopropyl $\beta-D-1$-thiogalactopyranoside; LB, Luria-Bertani medium; MIANS, 2-(4'-maleimidylanilino)naphthalene-6-sulfonic acid, sodium salt; Ni-NTA, nickel-nitrilotriacetic acid; NTD, N-terminal domain; PCR, polymerase chain reaction; PMSF, phenylmethylsulfonyl fluoride; RCF, relative centrifugal force; RNAPII, RNA polymerase II; SDS-PAGE, sodium dodecyl sulfate-polyacrylamide gel electrophoresis; TBP, TATA-box binding protein; TCEP, tris(2-carboxyethyl) phosphine; TCSPC, time-correlated single photon counting, TFIIB, general transcription factor IIB 
\& Roberts, 2005; Elsby et al., 2006; Tran \& Gralla, 2008; Zheng et al., 2004).

\section{MATERIALS AND METHODS}

Materials. Agarose was purchased from ABO. IPTG was from Biosynth AG. Bromophenol blue, DTT, $\beta$-mercaptoethanol, Nonidet P-40, PMSF, TCEP, Zinc chloride were from Fluka. Bisacrylamide, Coomassie Brillant Blue, Ethidium bromide, Acrylamide, SDS were from ICN. Ammonium persulfate, Glycerol, Magnesium chloride, TEMED were from BioShop. Agar and Tris were from MP Biomedicals. Ethanol, Methanol, Orthophosphoric acid, Potassium chloride, Sodium chloride, Acetic acid were from POCh. Ampicillin was from Polfa Tarchomin. Calcium chloride, EDTA, Glycine, Hepes, Imidazole, PEG8000, Isopropanol were from Sigma-Aldrich. Yeast extract was from Invitrogen. Unstained Protein Ladder, GeneRuler ${ }^{\mathrm{TM}} 1 \mathrm{~kb}$ DNA Ladder, $P f u$ polymerase, dNTPs, restriction enzymes: NheI, HindIII, DpnI and proper reaction buffers were purchased from Fermentas. Ni-NTA His-Bind Resin was from Novagen. Clean-Up, Gel-Out and Plasmid Mini kits were from A\&A Biotechnology. All measurements were performed in buffers prepared in water purified with the Millipore system.

Plasmids. pIIB1 encoding open reading frame of human TFIIB - gift of I. Ha and D. Reinberg (Ha et al., 1991); pET-21a (Novagen) — expression vector.

Primers. See Table 1.

Bacterial strains. E. coli DH5 $\alpha$ (Invitrogen) - used in cloning and for plasmid amplification; E. coli BL21(DE3) (Invitrogen) - used for TFIIB expression.

Oligonucleotide duplexes containing the DNA sequences specifically recognized by the TFIIB protein:

AdML

5' -AGGGGGGCCTATAAAAGGGGGTGGGG-3'

3'-TCCCCCCGGATATTTTCCCCCACCCC-5'

AdE4

5' -TTTTAGTCCTATATATACTCGCTCTG-3'

3' -AAAATCAGGATATATATGAGCGAGAC-5'

Cloning and site directed mutagenesis. The fulllength human TFIIB gene was amplified by polymerase chain reaction (PCR) from pIIB1 using $P f u$ polymerase (Fermentas). To create the TFIIB-His ${ }_{6}$ construct, TF2f and TF2r primers were used, and the gene was cloned into NheI and HindIII sites in the pET21a vector (Novagen) and sequenced. All cloning steps were performed using the E. coli DH5 $\alpha$ (Novagen) strain. The cloning protocol included the proteinase K (Sigma-Aldrich, molecular biology grade) digestion step (Crowe et al., 1991). For carrying out modification of the TFIIB-His ${ }_{6}$ construct, QuikChange (Stratagene) directed mutagenesis technique have been used in standard conditions. W52 residue has been substituted by phenylalanine, tyrosine, valine or leucine, using TF2W52Ff \& TF2W52Fr; TF2W52Yf \& TF2W52Yr; TF2W52Vf \& TF2W52Vr; TF2W52Lf \& TF2W52Lr primers respectively. In case of substitution of cysteine residues: C181S, C194S or C223S, TF2C181Sf \& TF2C181Sr; TF2C194Sf \& TF2C194Sr; TF2C223Sf \& TF2C223Sr; primers were used respectively. All cloning steps were performed using the E. coli DH5 $\alpha$ (Novagen) strain. All the obtained constructs were sequenced and aligned with human TFIIB sequence from NCBI database (accession code: NM_001514).

Protein expression and purification. For expression experiments, the plasmids were freshly transformed into the E. coli strain BL21(DE3) (Novagen) and were grown on the LB-agar plates with ampicillin $(100 \mu \mathrm{g} / \mathrm{mL})$ overnight at $37^{\circ} \mathrm{C}$. LB-ampicillin medium $(2 \mathrm{~mL})$ was inoculated with a single colony from LB-ampicillin agar plate and grown for $8 \mathrm{~h}$ at $37^{\circ} \mathrm{C}$, then this culture was used to inoculate $30 \mathrm{~mL} \mathrm{LB}$-ampicillin medium and grown at $37^{\circ} \mathrm{C}$. The overnight culture was added to $2 \mathrm{~L}$ of LBampicillin and grown in Erlenmeyer flask in water bath with $150 \mathrm{RPM}$ shaking at $37^{\circ} \mathrm{C}$. Expression was induced at an O.D. 600 of $0.5-0.6$ with $0.1 \mathrm{mM}$ isopropyl $\beta$-D-1thiogalactopyranoside (IPTG) overnight at $18^{\circ} \mathrm{C}$. At the time of induction, the cultures were supplemented with $\mathrm{ZnCl}_{2}$ to a concentration of $0.1 \mathrm{mM}$. Cells were harvested by centrifugation at $7000 \times g$ for $15 \mathrm{~min}$ at $4^{\circ} \mathrm{C}$, pelleted and stored frozen at $-20^{\circ} \mathrm{C}$.

The bacterial pellet from the $2 \mathrm{~L}$ culture was resuspended in $30 \mathrm{~mL}$ of buffer A (Hepes $50 \mathrm{mM} \mathrm{pH} \mathrm{7.5,}$ $\mathrm{NaCl} 200 \mathrm{mM}$, glycerol 20\%); buffer A contained also DNAse $100 \mathrm{U} / \mathrm{mL}, \mathrm{CaCl}_{2} 5 \mathrm{mM}, \mathrm{MgCl}_{2} 2.5 \mathrm{mM}$ and phenylmethylsulfonyl fluoride (PMSF) $1 \mathrm{mM}$. The suspension was sonicated for six 30-s bursts on ice. The lysate was cleared by centrifugation (20 krcf (relative centrifugal force), $60 \mathrm{~min}, 4^{\circ} \mathrm{C}$ ), and the supernatant was loaded onto a 5-mL nickel-nitrilotriacetic acid (Ni-NTA) gravity-flow column (Novagen), which was previously equilibrated in buffer $\mathrm{A}$ containing also $\mathrm{CaCl}_{2}$ $5 \mathrm{mM}, \mathrm{MgCl}_{2} 2.5 \mathrm{mM}$, PMSF $1 \mathrm{mM}$ and dithiothreitol (DTT) $1 \mathrm{mM}$. The column was washed with 15-20 column volumes (CV) of buffer A1 (Hepes $50 \mathrm{mM} \mathrm{pH} \mathrm{7.5,}$ $\mathrm{NaCl} 1 \mathrm{M}$, glicerol $20 \%$, imidazole $20 \mathrm{mM}$ and PMSF $1 \mathrm{mM}$ ) with addition of DTT $1 \mathrm{mM}$. The protein was then eluted in buffer A with addition of $300 \mathrm{mM}$ im-

Table 1. Sequences of primers used for cloning and site directed mutagenesis

\begin{tabular}{lll}
\hline Primer designation* & forward primers & reverse primers \\
\hline TF2 & CTAGCTAGCACCAGCCTGTTGG & CCCAAGCT TTAGCTGTGGTAG \\
\hline TF2W52F & GGATCTGA ATTCGAACTTC & GAAAGTT CG AAATTCAGATCC \\
\hline TF2W52Y & GGATCTGAATATCGAACTTC & GAAAGTCG ATATTCAGATCC \\
\hline TF2W52V & GGAT CTGA AGTGCGAACTTC & GAAAGTTCG CACTTCAGATCC \\
\hline TF2W52L & GGATCTGAATTGCGAACTTC & GAAAGTCG CAATTCAGATCC \\
\hline TF2C181S & CATT TAAA GAA ATATCTGCCGTATCACGAATTC & GAAATTCGT GATACGGCAGATATTCTTTAAATG \\
\hline TF2C194S & GAAAGAAA TTGGTCGGTCTTTAAACTTATTTG & CAAAATAA GTTAAAAGACCGACCAATTCTTC \\
\hline TF2C223S & GGTTCTGTTCCAACCTTAGCCTTCC & GGAAGGCT AAGGTTGGAACAGAACC \\
\hline
\end{tabular}

The sequences are given in order from $5^{\prime}$ to $3^{\prime} ;{ }^{*}$ For the primer names used in the text the letters " $\mathrm{f}^{\prime \prime}$ and " $\mathrm{r}^{\prime \prime}$ were added to denote the forward and reverse primers, respectively. 
idazole and $1 \mathrm{mM}$ DTT, $1.5 \mathrm{~mL}$ fractions were harvested. In case of samples prepared for fluorescent labeling, tris(2-carboxyethyl)phosphine (TCEP) substituted DT'T in the last $2 \mathrm{CV}$ of $\mathrm{A} 1$ wash and in the elution buffer. The whole purification procedure was carried at $4^{\circ} \mathrm{C}$ to decrease protease activity, thereby reducing protein digestion level. The protein concentration was determined in triplicates with the Bradford assay (Bradford, 1976), calibrated with bovine serum albumin (BSA) (Sigma-Aldrich). The purification process was monitored by sodium dodecyl sulfate-polyacrylamide gel electrophoresis (SDS/PAGE) (Laemmli, 1970) in 12\% gel. Fractions containing TFIIB or its variants were pooled and subjected to three-step dialysis at $4^{\circ} \mathrm{C}$ against buffer $\mathrm{A}$ with addition of DTT $5 \mathrm{mM}$ (1:100 ratio) or buffer B (Hepes $50 \mathrm{mM}$ pH 7.9, $\mathrm{NaCl} 100 \mathrm{mM}$, glycerol 10\%, DTT 2 $\mathrm{mM}$ ) and frozen at $-80^{\circ} \mathrm{C}$.

Fluorescence labeling. In order to covalently label the sulfhydryl groups of TFIIB or its variants, 2-(4'-maleimidylanilino)naphthalene-6-sulfonic acid (MIANS) (Molecular probes) was used. The dye stock solution was prepared in concentration of $20 \mathrm{mM}$ in $100 \%$ dimethyl sulfoxide (DMSO), and kept in darkness to protect the dye molecules from light. The protein was subjected to labeling directly after the purification procedure - before final dialysis. The Dye reagent was added in molar excess $(2: 1 ; 4: 1 ; 6: 1 ; 8: 1 ; 9: 1 ; 11: 1)$, taking care not to exceed $1 \%$ DMSO concentration. The mixtures were incubated for 30 minutes at room temperature in darkness, then DTT was added to a concentration of $1 \mathrm{mM}$. The unbound fluorescent dye reacted with DT'T and was then removed by the dialysis described above. After the third dialysis step, the protein labeling degree was calculated as the ratio of the absorption-determined concentration of the fluorescent probe, and the concentration of the protein determined by the Bradford method. The extinction coefficient used to calculate the concentration of MIANS according to the Beer-Lambert law was 27000 $\mathrm{M}^{-1} \mathrm{~cm}^{-1}$ at $322 \mathrm{~nm}$.

Spectroscopic studies. Circular dichroism measurements were conducted using a J-710 spectropolarimeter (Jasco) equipped with an F25 temperature control unit (Julabo) in quarts cuvettes of $0.2 \mathrm{~mm}$ path-length (Hell$\mathrm{ma}$ ). The spectra were collected in the range of 190-250 $\mathrm{nm}$ with $1 \mathrm{~nm}$ data pitch, $10 \mathrm{~nm} / \mathrm{min}$ scanning speed, $8 \mathrm{~s}$ integrating time, and $2 \mathrm{~nm}$ bandwidth and averaged over three acquisitions.. All spectra were corrected for the effect from the buffer and all measurements were converted to molar residual ellipticity units as described elsewhere. The protein concentration were $20 \mu \mathrm{M}$.

Fluorescence emission spectra were recorded in buffer A using spectrofluorimeter Fluorolog-3 equipped with an F25 temperature control unit (Julabo). Spectra were measured in thermostated cuvette $3 \times 3 \mathrm{~mm}$ (Hellma) at $25^{\circ} \mathrm{C}$. Spectra were obtained with a $1 \mathrm{~nm}$ data pitch, integration time $0.2 \mathrm{~s}$ for every wavelength and averaged over three acquisitions. In case of the dye fluorescence measurements excitation wavelength was $320 \mathrm{~nm}$ and range of emission spectrum was $350-500 \mathrm{~nm}$. Slit widths on excitation and emission monochromators were set at 3 and $2 \mathrm{~nm}$ respectively, for TFIIB wt, TFIIB C194S and TFIIB C194S C223S, or 3 and $3 \mathrm{~nm}$, respectively for C181S or 4 and $3 \mathrm{~nm}$ respectively for C223S mutants. In every case, buffer A spectrum was first measured and then subtracted from sample measurement spectrum. The protein concentration was $1 \mu \mathrm{M}$, while ds DNA (AdML or AdE4) concentration was 0.5, 1, 1.5 and $2 \mu \mathrm{M}$ (if present).
The steady-state fluorescence quenching experiments of TFIIB intrinsic fluorescence (tryptophan residue W52) were performed at $25^{\circ} \mathrm{C}$ in buffer $\mathrm{B}$ in a Fluorolog-3 equipped with an F25 temperature control unit (Julabo). The spectra were measured in thermostated quartz cuvette $10 \times 4 \mathrm{~mm}$ (Hellma) at $25^{\circ} \mathrm{C}$. The corrected spectra were recorded in the range from 305 to $450 \mathrm{~nm}$, with $0.5 \mathrm{~nm}$ data pitch, integration time $0.1 \mathrm{~s}$ for every wavelength and averaged over three acquisitions. The excitation wavelength was $295 \mathrm{~nm}$. The excitation and emission slits were set at 5 and $5 \mathrm{~nm}$, respectively. Four molar (4 M) KI solution used as a fluorescence quencher contained $0.1 \mathrm{M}$ sodium thiosulfate to inhibit the formation of $\mathrm{I}_{3}^{-}$. Fluorescence intensities were corrected for any dilution effects as well as for residual buffer emission and Raman scattering. The quenching data were analyzed using the Stern-Volmer equation:

$$
\frac{F}{F_{0}}=\sum_{i} \frac{f_{i}}{1+K_{i}[Q]}
$$

where $\mathrm{F}_{0}$ and $\mathrm{F}$ are fluorescence intensities in the absence and presence of quencher, Q, respectively, $K_{\mathrm{i}}$ is the dynamic quenching constant and $f_{i}$ is the fractional contribution of component $i$. The quenching rate constant $k_{\mathrm{q}}$ was calculated as $k_{\mathrm{q}}=K_{\mathrm{i}} /\langle\tau\rangle$, where $\langle\tau\rangle$ is the mean fluorescence lifetime in the absence of the quencher. The Stern-Volmer equation was fitted to the experimental data by an iterative nonlinear least square method using Origin 9.1 software. The data were analyzed using one-way analysis of variance (ANOVA) test (GraphPad Prism 5.0, USA) with the t-test to compare the statistical significance between groups. A p-value of less than 0.05 indicated statistically significant results.

Time-correlated single photon counting (TCSPC) measurements were performed on a FluoroCube fluorimeter (Horiba, France). Tryptophan residue was excited with pulsed NanoLED diodes at $294 \mathrm{~nm}$ (pulse duration of $700 \mathrm{ps}$ ) operated with $1 \mathrm{MHz}$ repetition. Maximum number of photons to be counted was set to 10000 and in a 200 ns range. To avoid pulse pile-up, the power of the pulses was adjusted to an appropriate level using a neutral gradient filter. The fluorescence emission was recorded using a picosecond detector TBX04 (IBH, JobinYvon, $\mathrm{UK})$ data station and the DAS 6 (JobinYvon (IBH, UK)) software was used for data acquisition and signal analysis. All the fluorescence decays were measured in a $10 \times 10$ $\mathrm{mm}$ quartz cuvette, using emitter cut-off filter with transmittance $>320 \mathrm{~nm}$ (320FG01 glass filter from and Andover corp.) The excitation profiles, required for the deconvolution analysis, were measured without the emitter filters on a light scattering cuvette. All measurements were performed at $25^{\circ} \mathrm{C}$. Each fluorescence decay was analyzed with a multi-exponential model given by the equation:

$$
I_{t}=\sum_{i} \alpha_{i} \exp \left(\frac{t}{\tau_{i}}\right)
$$

where $\alpha_{\mathrm{i}}$ and $\tau_{\mathrm{i}}$ are the pre-exponential factor and decay time of component $i$, respectively. Best fit parameters were obtained by minimization of reduced $\chi^{2}$ value as well as residual distribution of the experimental data. The mean lifetime of fluorescence decay was calculated according to the following equation:

$$
\langle\tau\rangle=\frac{\sum_{i} \alpha_{i} \tau_{i}^{2}}{\sum_{i} \alpha_{i} \tau_{i}}
$$




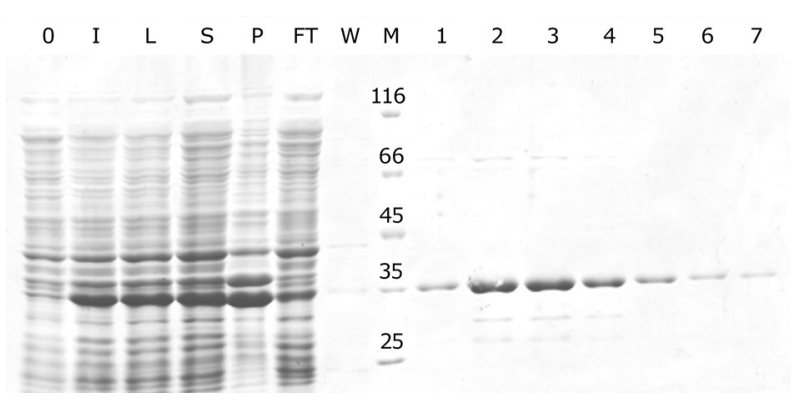

Figure 1. SDS/PAGE analysis of TFIIB purification process. 0 - bacterial culture prior to induction; I - bacterial culture after the induction; $\mathrm{L}-$ bacterial cell lysate; $\mathrm{S}$ - supernatant obtained after centrifugation; P - pellet; FT - NiNTA flowthrough; $\mathrm{W}-$ NiNTA wash (20 mM imidazole); $M$ - molecular mass marker; 1-7 - fractions eluted from NiNTA with $300 \mathrm{mM}$ imidazole. The numbers on the marker lane indicate protein molecular mass in $\mathrm{kD}$.

\section{RESULTS}

\section{Protein expression and purification}

Recombinant human TFIIB was produced in pET expression system with $\mathrm{His}_{6}$-tag at its $\mathrm{C}$-terminus and constituted the majority of the total protein pool (Fig. 1, lanes I, L). SDS/PAGE analysis also showed that the amount of TFIIB in soluble and insoluble fractions (denoted as $\mathrm{S}$ and $\mathrm{P}$ in the Fig. 1) are comparable. The protein was purified from the soluble fraction to high homogeneity, above $95 \%$, with use of metal affinity chromatography (Fig. 1, lanes 1-7). The purity was adequate for structural studies. Similar condition for TFIIB

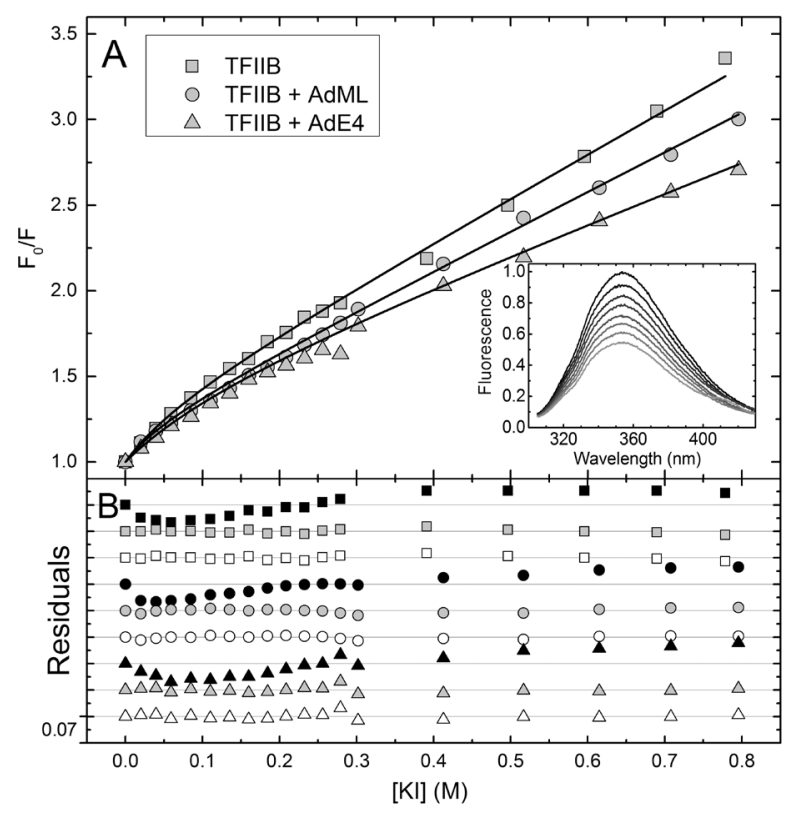

Figure 2. Quenching of TFIIB intrinsic fluorescence by iodide. (Panel A) Stern-Volmer plot for TFIIB alone (squares), and in the presence of AdML (circles) and AdE4 (triangles). Continuous lines represent the best fits to the experimental data. Insert: a representative set of TFIIB emission spectra in the absence of DNA obtained for KI concentrations: $0.00 \mathrm{M} ; 0.01 \mathrm{M} ; 0.03 \mathrm{M} ; 0.05 \mathrm{M} ; 0.08$ $\mathrm{M} ; 0.11 \mathrm{M} ; 0.18 \mathrm{M}$; and 0.23 M. (Panel B) Residual distribution in term of one-component (black) two-component (gray) and threecomponent (open) analysis of Stern-Volmer equation. expression and purification has been reported previously (Thompson et al., 2009; Thomsen et al., 2001).

\section{Intrinsic fluorescence studies}

TFIIB exhibited intrinsic fluorescence due to its single tryptophan residue, located in the hinge region that connects NTD and CTD. TFIIB fluorescence emission was susceptible to quenching with iodide.Typical Stern-Volmer plots in the absence and in the presence of specific DNA are presented in Fig. 2.

In each case the Stern-Volmer plots curved down and could not be satisfactorily fitted to a single-component model, i.e. to Eqn. (1) with $\mathrm{n}=1$. Addition of a second component significantly improved the fit, while addition of a third component did not improve it further. In case of the apo protein, the obtained reduced $\chi^{2}$ values were: $1.02 \times 10^{-3}, 3.00 \times 10^{-5}$ and $3.40 \times 10^{-5}$ for the single-, double- and triple-component analysis, for AdML-ligated TFIIB these values equaled: $9.04 \times 10^{-4}$, $3.38 \times 10^{-5}, 2.19 \times 10^{-5}$ and for AdE4 ligated protein $1.13 \times 10^{-4}, 6.01 \times 10^{-5}$ and $6.87 \times 10^{-5}$. The $\chi^{2}$ values indicated that in each case the triple-component analysis was not significantly better than the double-component analysis. Also, in any case the distribution of the residuals was not improved upon the addition of the third exponent (Fig. 2B). Therefore the fluorescence quenching can be described by a two-component model, in which one fluorophore class is less accessible for the quencher and characterized by $K_{1}=1.83 \pm 0.14 \mathrm{M}^{-1}$ for the apo protein, while the other is more accessible for iodide with $K_{2}=16.19 \pm 2.77 \mathrm{M}^{-1}$. The presence of the specific DNA sequence changed the value of the first component slightly if any, to $1.83 \pm 0.11 \mathrm{M}^{-1}$ and $1.12 \pm 0.19 \mathrm{M}^{-1}$ for AdML and AdE4, respectively. The value of the quenching constant for the second component varied more significantly in the presence of a specific DNA and was equal to $21.03 \pm 4.56 \mathrm{M}^{-1}$ and $9.69 \pm 1.98 \mathrm{M}^{-1}$ for AdML and AdE4, respectively.

To further analyze the quenching parameters, timeresolved measurements were performed. Figure 3 shows an exemplary TFIIB intrinsic fluorescence decay.

The best fit was obtained for triple exponential decay, giving decay times of $0.50 \pm 0.05 \mathrm{~ns}, 2.86 \pm 0.07$ $\mathrm{ns}, 6.53 \pm 0.09 \mathrm{~ns}$, with respective contributions: 0.11 $\pm 0.01,0.55 \pm 0.01,0.34 \pm 0.01$. The mean fluorescence lifetime, calculated from equation 3 , was $3.85 \pm$

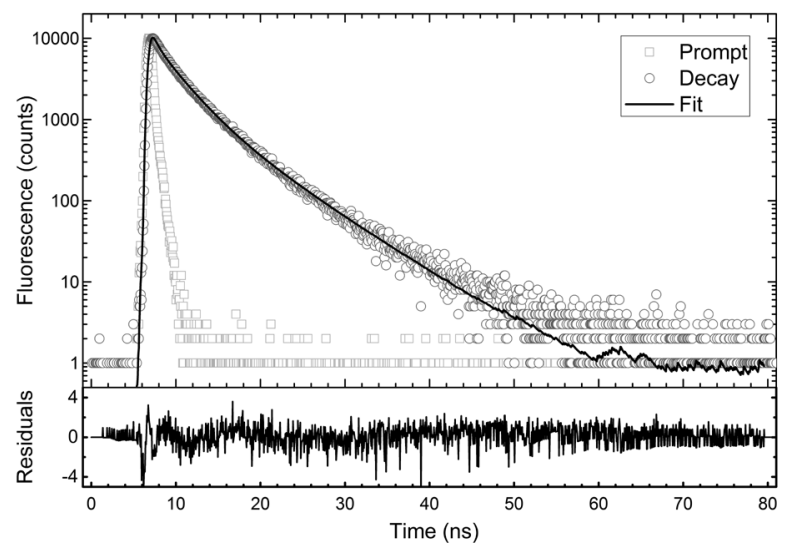

Figure 3. Fluorescence lifetime of TFIIB.

(Upper panel) Open circles show the decay of W52 fluorescence emission (Decay), while solid lines are a triple exponential fit. The excitation pulse profile, set up at $294 \mathrm{~nm}$ is show by the squares curve (Prompt). (Bottom panel) residual distribution for the fitting of three exponents. 
Table 2. Fluorescence quenching parameters for TFIIB and its DNA complexes.

\begin{tabular}{llll}
\hline species & $\mathrm{f}_{1}$ & $k_{\mathrm{q} 1}\left(10^{9} \mathrm{M}^{-1} \mathrm{~s}^{-1}\right)$ & $k_{\mathrm{q} 2}\left(10^{9} \mathrm{M}^{-1} \mathrm{~s}^{-1}\right)$ \\
\hline TFIIB & $0.69 \pm 0.04$ & $0.48 \pm 0.04$ & $4.2 \pm 0.7$ \\
\hdashline TFIIB + AdML & $0.78 \pm 0.03^{\mathrm{a}}$ & $0.48 \pm 0.03$ & $5.5 \pm 1.2$ \\
\hdashline TFIIB + AdE4 & $0.61 \pm 0.07^{\mathrm{b}}$ & $0.29 \pm 0.05^{\mathrm{a}, \mathrm{bb}}$ & $2.5 \pm 0.5^{\mathrm{a}, \mathrm{b}}$ \\
\hline
\end{tabular}

The statistical significance was evaluated using one-way ANOVA, followed by T-test comparisons. The following symbols were used: a $P<0.05$; aa $P<0.01$ for the comparison of apo TFIIB with TFIIB ligated with DNA, ${ }^{b} P<0.05$; ${ }^{b} P<0.01$ for the comparison of TFIIB ligated with AdML vs ligated with AdE4.

$0.02 \mathrm{~ns}$ and is typical for tryptophan residues. Quenching rate constants were calculated from the obtained values and presented in Table 2 with the fractions of the individual components. One-way ANOVA showed statistical differences for $\mathrm{f}_{1}[\mathrm{~F}(2.8)=8.797 ; \quad P<0.05]$; $k_{\mathrm{q} 1}[\mathrm{~F}(2.8)=21.66 ; P<0.01] ;$ and $k_{\mathrm{q} 2}[\mathrm{~F}(2.8)=9.344$; $P<0.05]$. T-test was used to compare statistical difference between the groups.

Statistical analysis showed that the parameters $k_{\mathrm{q} 1}$ and $k_{\mathrm{q} 2}$ changed in a statistically significant way only for TFIIB bound to AdE4 sequence. The analysis showed also a statistically significant change in the parameter $f_{1}$, although it concerned only the case when TFIIB was associated with AdML sequence. Thus, all three parameters are different for TFIIB after binding to AdML and AdE4 promoters.

\section{Changing the fluorescent properties of TFIIB}

In order to modify the fluorescent properties of TFIIB, W52 was replaced with other residues: phenylalanine, tyrosine, leucine, or valine, using site directed mutagenesis. Despite obtaining the correct genetic constructs, very low level of protein expression precluded their purification in the case of substitutions with leucine and valine (data not shown). Introduction of aromatic residues in place of W52 did not cause any significant decrease in the level of expression, although the circular dichroism spectra of these proteins (Fig. 4) showed that the secondary structure has been changed.

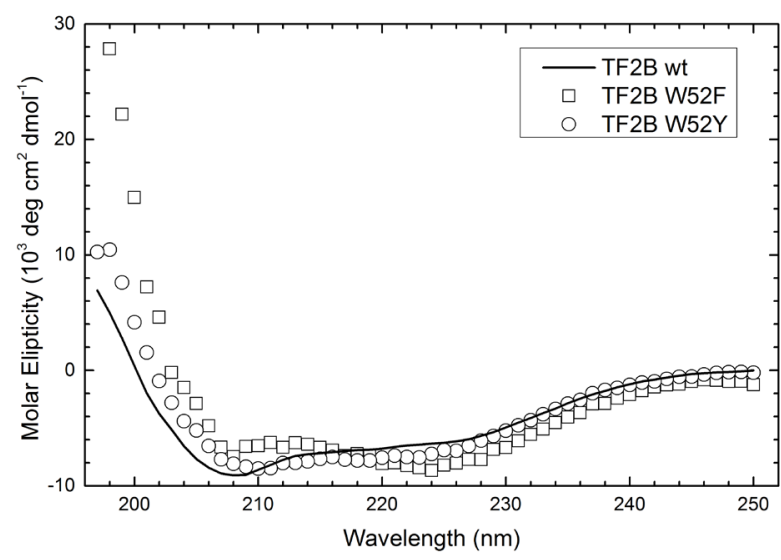

Figure 4. Circular dichroism spectra of TFIIB mutants with substitutions of $W 52$ residue.

Solid line - wild-type TFIIB; squares - TFIIB W52F; circles - TFIIB W52Y. The obtained results indicate significant structural importance of W52 residue. An alignment of amino acid sequence of several TFIIB homologs was conducted using Clustal Omega program (Sievers et al., 2011). Fragments of the alignment are shown in the upper part of Fig. 5.

The result presented above proves the high evolutionary conservation of W52 residue. As we were unable to substitute it without interfering with the protein structure, we decided to modify the fluorescent properties of TFIIB by fluorescent labeling. A solvatochromic dye, MIANS, was used, which covalently binds to sulfhydryl groups. Due to the presence of three water-accessible cysteine residues within the cyclin-like repeats of CTD, a series of TFIIB variants was designed to make the labeling more specific. None of these residues is evolutionarily conserved, as can be judged by the alignment of TFIIB homolog sequences presented in the bottom part of Fig. 5. Therefore each of them was substituted, i.e., C181S, C194S, C232S, and additionally a double mutant C194S C232S was prepared. None of the mutations altered the protein secondary structure (Fig. 6).

\section{Extrinsic fluorescence studies}

MIANS emission intensity depends on the polarity of its microenvironment. The emission spectra col-

20

30

40

52

60

70

80

TF2B HUMAN PDAILVEDYRAGDMICPECGLVVGDRVIDVGSEWRTFSNDK-ATKDPSRVGDSQNPLLSDGDLSTMIGKG TF2B_RAT PDAILVEDYRAGDMICPECGLVVGDRVIDVGSEWRTFSNDK-ATKDPSRVGDSQNPLLSDGDLSTMIGKG TF2B XENLA PDALLVEDYRAGDMICSECGLVVGDRVIDVGSEWRTFSNDK-AAADPSRVGDAQNPLLSGGDLTTMIGKG TF2B DROME PESPLIEDYRAGDMICSECGLVVGDRVIDVGSEWRTFSNEK-SGVDPSRVGGPENPLLSGGDLSTIIGPG TF2B_YEAST YPPKIVERFSEGDVVCALCGLVLSDKLVDTRSEWRTFSNDDHNGDDPSRVGEASNPLLDGNNLSTRIGKG TF2B_PYRFU ESAELIYDPERGEIVCAKCGYVIEENI IDMGPEWRAFDASQ--RERRSRTGAPESILLHDKGLSTEIGITF2B SULSH PPDKIIFDAERGEYICSETGEVLEDKIIDQGPEWRAFTPEE--KEKRSRVGGPLNNTIHDRGLSTLIDWK

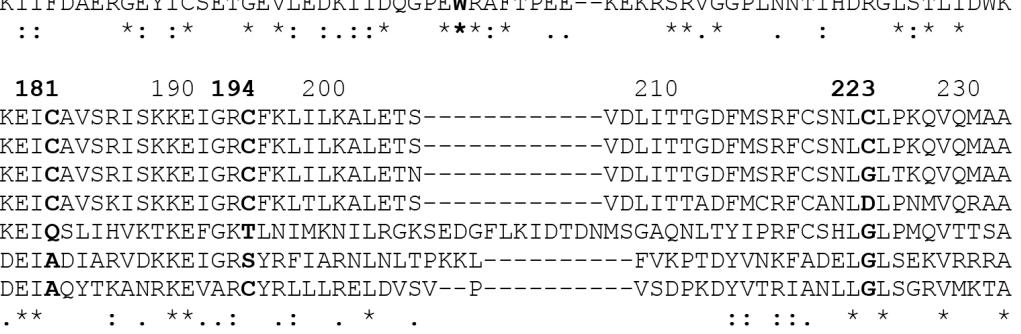

Figure 5. Alignment of fragments of TFIIB sequence for homologs from: Homo sapiens, Rattus norvegicus, Xenopus leavis, Drosophila melanogaster, Saccharomyces cerevisiae, Pyrococcus furiosus, and Sulfolobus shibatae.

The last digit of the number in the first line indicates the number of amino acid residue in the human TFIIB. Bold letters indicate amino acid residues modified in the present study and the corresponding amino acid residues in the homologs. Stars indicate residues that are conserved among all the species, colon — in most of the species, dot — similar residues. Data was obtained using Clustal Omega program (Sievers et al., 2011). 


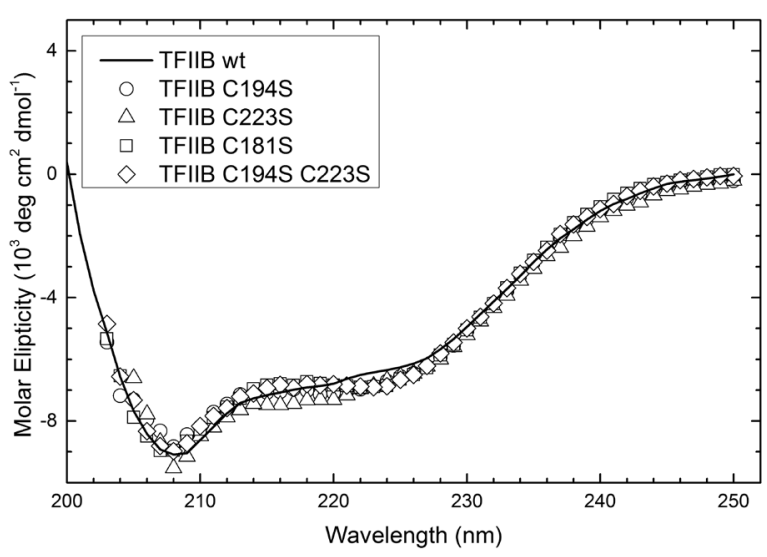

Figure 6. Circular dichroism spectra of TFIIB mutants with substitutions of cysteine residues.

Solid line - wild-type TFIIB; circles - TFIIB C194S; triangles - TFIIB C233S; squares - TFIIB C181S; rhombs - TFIIB C194S C233S. Labeling with MIANS was performed for the wild-type protein and the cysteine-depleted mutants, using several amounts of the dye (Fig. 7).

lected for MIANS-labeled proteins changed in the presence of AdML and AdE4, and the course of the abovementioned changes adopted a shape of a binding curve (Fig. 8). In case of the wild-type TFIIB, binding to DNA decreased MIANS fluorescence intensity only slightly, more significantly for AdE4. For the other protein variants, the changes were more profound. For C181S mutant, the observed increase in fluorescence intensity was independent of the recognized sequence, while for the C194S mutant a change was seen only upon binding to AdE4. Lack of C232 residue increased MIANS fluorescence intensity. The presence of DNA resulted in an increase in fluorescence intensity, higher for AdML than for AdE4. On the other hand, substitution of C194 residue resulted in a decrease in MIANS fluorescence intensity, which was in turn greater for AdE4 sequence. The maximum changes in fluorescence intensity for each case are presented in Table 3. T-test was used to compare the statistical difference between the groups.

\section{DISCUSSION}

Transcription factor IIB belongs to the family of general transcription factors and plays a crucial role in RNA polymerase II preinitiation complex assembly. Its existence as a monomer in solution, and lack of post-translational modifications necessary for its activity, makes TFIIB the only general transcription factor that may be obtained in the fully functional form in a prokaryotic expression system (Deng \& Roberts, 2007). These features make TFIIB especially attractive for studying the influence of other macromolecules on the protein structure and its dynamics, especially using fluorescence techniques.

Tryptophan fluorescence has been widely used as a sensitive probe for studying conformational changes in protein structure (Lakowicz, 2006). TFIIB exhibits intrinsic fluorescence due to W52 residue, located in the hinge region that connects NTD and CTD (Bangur et al., 1999). Both, the presence of the only one tryptophan residue and its location allowed us to monitor possible changes in TFIIB structure without any additional modification of the protein. The presence of iodide in the solution decreased the intrinsic fluorescence intensity, due

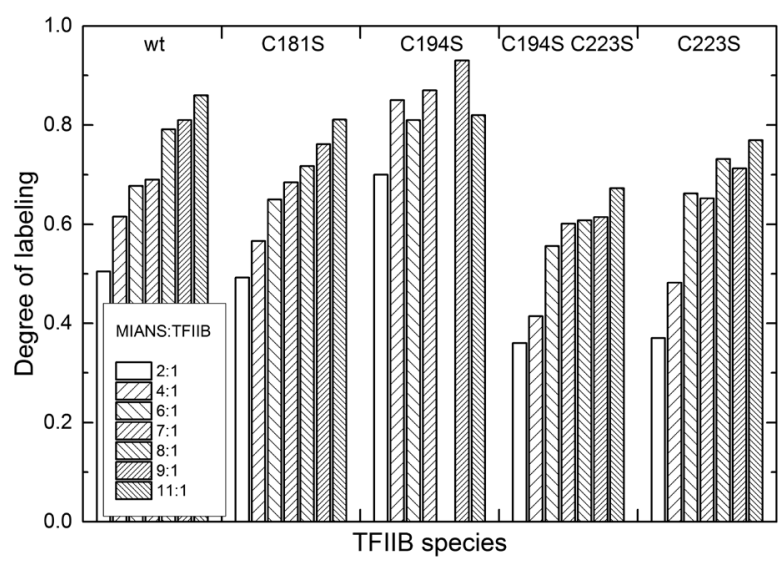

Figure 7. Degree of MIANS labeling (a label:protein ratio) for different TFIIB mutants, depending on the initial dye-protein stoichiometry.

Table 3. Relative fluorescence intensity change induced by the presence of DNA

\begin{tabular}{lcc}
\hline species & AdML & AdE4 \\
\hline TFIIB wt & $-6 \% \pm 1 \%$ & $-12 \% \pm 1 \%$ aa \\
\hline TFIIB C181S & $17 \% \pm 4 \%$ & $19 \% \pm 1 \%$ \\
\hline TFIIB C194S & $3 \% \pm 1 \%$ & $26 \% \pm 4 \%$ aaa \\
\hline TFIIB C223S & $88 \% \pm 10 \%$ & $63 \% \pm 4 \%$ a \\
\hline TFIIB C194S C223S & $-34 \% \pm 4 \%$ & $-54 \% \pm 5 \%$ aa \\
\hline
\end{tabular}

The statistical significance was evaluated using $T$-test for the comparison between AdE4 and AdML. The following symbols were used: a $P<0.05 ;$ aa $P<0.01 ;$ aaa $P<0.001$.

to effective collisional quenching of tryptophan fluorescence by the ion. The effectiveness of the quenching is determined in general by the extent of tryptophan exposure to the solvent, and typically for a single fluorophore it is described by linear relationship on the Stern-Volmer plot. Nevertheless, the graph we obtained was not linear but curved down, as can be seen in the Figure 2 . The phenomenon can be best described by a two-component model, with two fluorophore species differing in their availability for the quencher. For a single-tryptophan protein, this justifies the existence of two forms of the protein. This can be interpreted as occurrence of two conformations of TFIIB, as we verified the protein sample to be highly pure, stable and homogeneous by size-exclusion chromatography and thermal denaturation analysis (results not shown). The obtained result is consistent with the previously observed interaction between NTD and CTD, leading to coexistence of "open" and "closed" conformations of the protein in equilibrium (Elsby \& Roberts, 2004; Reese, 2003). The assumption that reported in the literature conformational states are identical to the conformational states observed in this study appears to be reasonable and allows to conclude that the quenching results indicate that these conformations differ significantly in W52 exposure to the solvent and remain in thermodynamic equilibrium.

The presence of DNA changed the quenching parameters, as can be seen in the Stern-Volmer plot (Figure 2). Moreover, AdML and AdE4 differently influenced the quenching efficiency. Thus the protein structure changed 

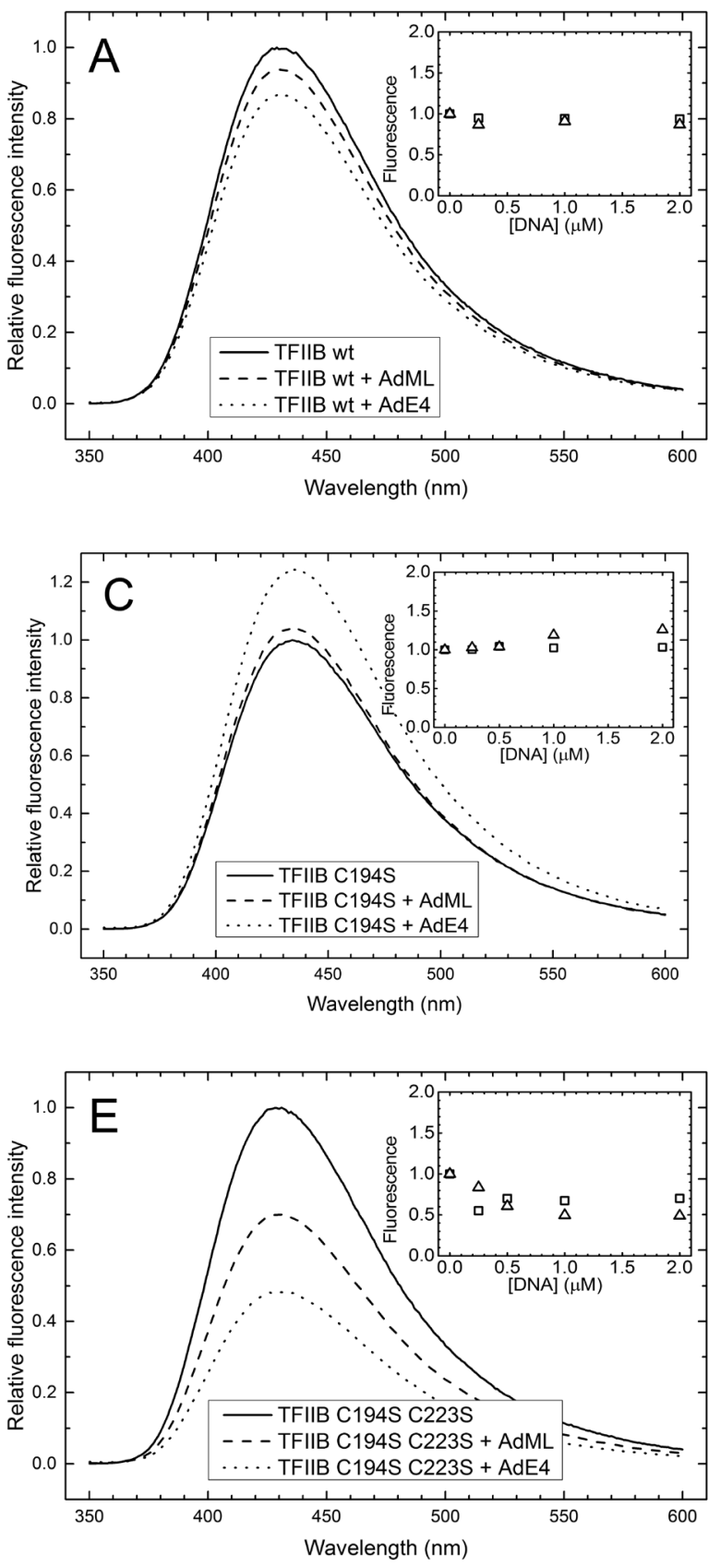

upon binding to DNA in a sequence-specific manner. The average fluorescence lifetime in the absence of the quencher served to calculate the bimolecular quenching rate constants shown in Table 2. In each case two fluorophore classes were observed, significantly differing in their accessibility to the quencher. The value of $k_{\mathrm{q} 1}$ indicates some level of fluorophore shielding, while $k_{\mathrm{q} 2}^{\mathrm{q} 1}$ value is close to the diffusion-controlled limit (Lakowicz, 2006). It is interesting that binding of AdML to TFIIB does not change any of the quenching rates characteristic for the two states, but only the equilibrium between the "open" and "closed" form. Therefore the presence of the DNA does not change the vicinity of W52 in these forms. Binding of AdML increases the value of the $f_{1}$ parameter, indicating greater dominance of the conformation with shielded tryptophan residue. In contrast, binding of AdE4 does not affect significantly the equilibrium between the two forms, but substantially shields
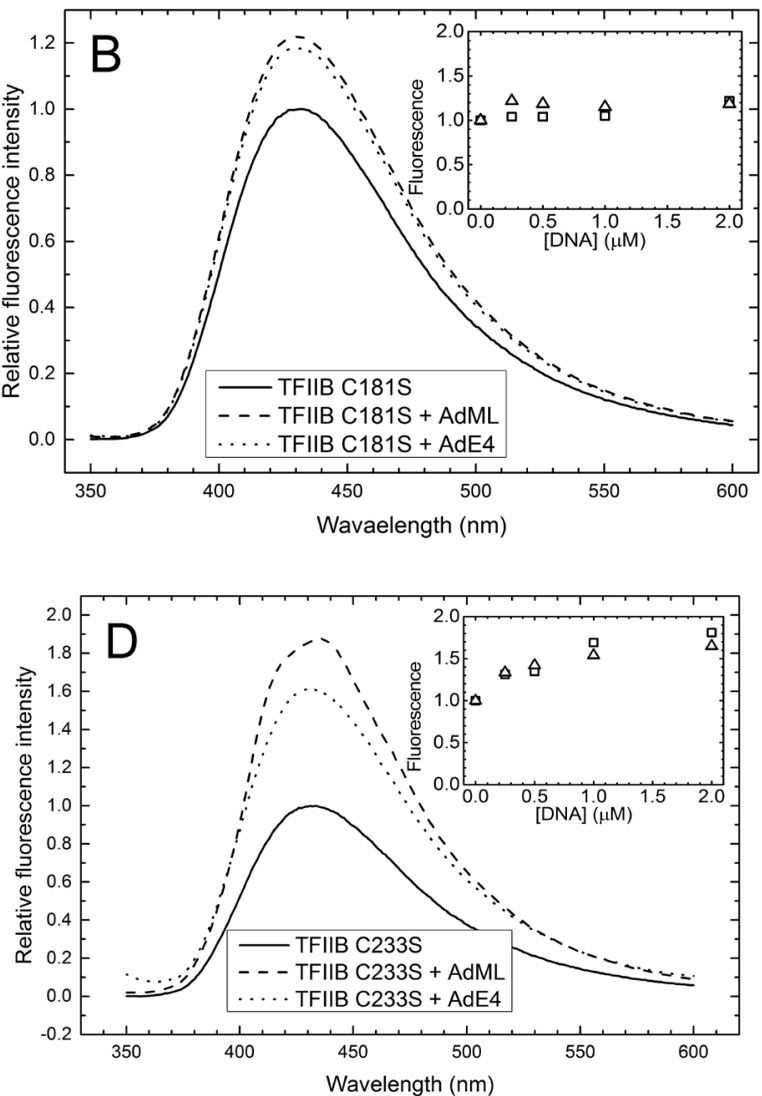

Figure 8. Emission spectra of MIANS for labeled TFIIB variants: (A) wild-type; (B) C181S; (C) C194S; (D) C223S; (E) C194S C223S. Lines represent the spectra obtained for the respective protein alone (solid lines), and in the presence of AdML (dashed lines) and AdE4 (dotted lines). The inserts show relative fluorescence intensity depending on the concentration of AdML (squares) or AdE4 (triangles).

the tryptophan residue in both of them. It means that W52 is more buried, or electrostatic repulsion renders the fluorophore less accessible to the quencher.

W52 is located in an important fragment of TFIIB that penetrates RNAPII during the formation of the preinitiation complex. The obtained results indicate that the microenvironment of the residue depends on the recognized promoter, and so is the interaction interface with RNAPII. It seems that TFIIB has the ability to "read" the sequence and affect the RNAP in a sequence-dependent manner.. It seems reasonable to draw the conclusion that in the transcription initiation process controlled by TFIIB, the DNA sequence is also important, apart from the equilibrium constants for interactions of the various transcription factors and DNA.

During the transcription activation, the transcription factors interact not only with RNAP, but also with each other, and therefore we decided to monitor other con- 
formational changes occurring in TFIIB. Since these interactions include also regions within CTD (i.e. interacting with VP16) we intended to relocate there the tryptophan residue as a fluorescent probe. This two-step process involved replacing W52 with a similar residue and introducing a tryptophan residue in the desired location. Most frequently, tryptophan residues are replaced by other aromatic or hydrophobic amino acid residues. W52 substitution with other aromatic residues influenced the protein secondary structure content (Fig. 4), while substitution with hydrophobic residues prevented the efficient expression. W52 is located within the most conserved region of TFIIB and is unchanged among eucaryal and archeal homologs (Fig. 5). The important functional role of W52 is also indicated by W52A substitution, which decreased TFIIB affinity to the viral protein Vpr (Agostini et al., 1999). Since it was impossible to relocate the tryptophan residue without disrupting the protein structure, we can assume that this residue plays not only a functional, but also a structural role, although it is located in the hinge region.

Due to the above-mentioned conclusions, the only possibility to monitor CTD structural changes with fluorescence measurements was to introduce an exogenous fluorophore, MIANS, that binds to sulfhydryl groups.Human TFIIB contains nine Cys residues, three of which form the zinc finger and are therefore inaccesible to chemical modification. Out of the remaining six cysteines, three are solvent-exposed: C181, C194, and C223 (Bagby et al., 1995) and therefore might be prone to labeling. All the three residues belong to the DNA-binding motifs but do not contact DNA directly (Nikolov et al., 1995). We substituted each of those cysteines with serine to make the labeling more specific, and to verify if they react with MIANS in the wild-type protein. The selected residues are not evolutionarily conserved (Fig. 5), so their substitution may have not affected the protein structure. The actual lack of structural significance of these residues was confirmed by circular dichroism spectroscopy (Fig. 6).

The obtained degree of labeling was higher for the wild-type TFIIB and lower for the mutants, confirming the initial assumptions concerning the reactivity of the particular cysteine residues. The degree of labeling correlated with the amount of dye added, but did not exceed 1.0 in any case. Both C194 and C223 seem to react with the dye, since C194S C223S mutant was modified to a lesser degree than both C194S and C223S. Labeling of C181S mutant was similarly effective compared to the wild-type protein. This suggests that C181 residue does not bind MIANS in the wild-type protein, although it might do so in the mutants bearing substitutions of the more reactive cysteine residues (e.g. C194S C223S). Thus the labeling was semi-specific and at least some of the dye was placed within the DNA-binding motifs.

The use of a solvatochromic fluorophore allowed to monitor structural changes induced by DNA binding in the vicinity of the dye. The emission spectra collected for MIANS-labeled proteins changed in the presence of DNA. The most profound changes were observed for the mutants lacking C223 (Fig. 8): twofold increase in MIANS fluorescence intensity was observed for TFIIB C223S and twofold decrease for TFIIB C194S C223S (Table 3). The observed signal changes significantly between the two promoter sequences and the difference was found statistically significant for all the protein variants except C181S. This could be explained by a different impact of AdML and AdE4 on the microenvironment of MIANS molecule bound to C181, localized within the BREd-binding domain while C194 and C223 contact BREu more closely. Since the observed changes varied significantly between the two promoter sequences, they cannot be attributed simply to the more hydrophobic milieu of the DNA major groove, but rather they manifest an alteration of the protein conformation that depends on the promoter sequence.

Interestingly, a stronger effect on the fluorescence intensity was observed for binding to AdE4 than to AdML - contrary to what could be expected from the fact that AdML is closer to the consensus sequence. A similar result was obtained by Zheng et al. in studies employing FRET between two fluorescent proteins fused to the ends of TFIIB molecule (Zheng et al., 2004). Different TFIIB epitopes are exposed during binding to AdML and to AdE4 (Fairley et al., 2002). AdE4 was also shown to be more sensitive than AdML to TFIIB mutation (Hawkes \& Roberts, 1999). Possibly, this difference might be caused by an interplay between BREu and BREd binding by TFIIB. Thus the observed differences probably reflect sequence-specific TFIIB action rather than simple difference in its affinity for the two sequences.

Our findings, which are consistent with the results obtained for prokaryotic transcription factor cAMP receptor protein (Fic et al., 2007), may lead to a more general conclusion. In the transcription initiation process, subtle changes in the structure and dynamics of the various transcription factors, depending on reciprocal interactions, play an important role, apart from the obvious structural aspects of the interaction between transcription factors and the RNAP, leading to suitable geometry of the complex formation, mutual affinity of the individual components or the kinetics of their interactions. These small changes can lead to significant synergies in the binding of these factors. Although this work is not an evident proof of the existence of such a process, it strongly suggests the possibility and the universality of its occurrence.

\section{Acknowledgements}

The Faculty of Biochemistry, Biophysics and Biotechnology is a partner of the Leading National Research Center (KNOW) supported by the Ministry of Science and Higher Education.

This research was supported by grant number 3128/P01/2006/31 from the Polish Ministry of Science and Higher Education.

We would like to thank Ola Kalużny and Damian Dawidowski for their help during the preparation of the plasmid constructs and preliminary studies, and Agata Faron-Górecka for her assistance during statistical analysis.

\section{Declaration of interest}

The authors declare no financial or personal interest with other people or organizations that could bias their work.

\section{REFERENCES}

Agostini I et al. (1999) The HIV-1 Vpr co-activator induces a conformational change in TFIIB. FEBS Lett 450: 235-239.

Bagby S et al. (1995) Solution structure of the C-terminal core domain of human TFIIB: similarity to cyclin A and interaction with TATAbinding protein. Cell 82: 857-867.

Bangur CS et al. (1999) An interaction between the N-terminal region and the core domain of yeast TFIIB promotes the formation of 
TATA-binding protein-TFIIB-DNA complexes. I Biol Chem 274: 23203-23209.

Bradford MM (1976) A rapid and sensitive method for the quantitation of microgram quantities of protein utilizing the principle of proteindye binding. Anal Biochem 72: 248-254.

Chen HT et al. (2000) Structure of a (Cys3His) zinc ribbon, a ubiquitous motif in archaeal and eucaryal transcription. Protein Sci: Publication of the Protein Society 9: 1743-1752.

Crowe JS et al. (1991) Improved cloning efficiency of polymerase chain reaction (PCR) products after proteinase $\mathrm{K}$ digestion. Nucleic Acids Res 19: 184.

Deng W, Roberts SG (2005) A core promoter element downstream of the TATA box that is recognized by TFIIB. Genes Develop 19: 2418-2423.

Deng W, Roberts SG (2007) TFIIB and the regulation of transcription by RNA polymerase II. Chromosoma 116: 417-429.

Elsby LM et al. (2006) Assembly of transcription factor IIB at a promoter in vivo requires contact with RNA polymerase II. EMBO Reports 7: 898-903.

Elsby LM, Roberts SG (2004) The role of TFIIB conformation in transcriptional regulation. Biochemi Soc Trans 32: 1098-1099.

Fairley JA et al. (2002) Core promoter-dependent TFIIB conformation and a role for TFIIB conformation in transcription start site selection. Mol Cell Biol 22: 6697-6705.

Fic E, Górecki A, Wasylewski Z (2007) Fluorescence quenching studies of conformational changes induced by cAMP and DNA binding to heterodimer of cyclic AMP receptor protein from Escherichia coli. Protein J 26: 457-466.

Grossmann JG et al. (2001) Molecular shapes of transcription factors TFIIB and VP16 in solution: implications for recognition. Biochemistry 40: 6267-6274.

Ha I, Lane WS, Reinberg D (1991) Cloning of a human gene encoding the general transcription initiation factor IIB. Nature 352: 689-695.

Hawkes NA, Evans R, Roberts SG (2000) The conformation of the transcription factor TFIIB modulates the response to transcriptional activators in vivo. Curr Biol: CB 10: 273-276.

Hawkes NA, Roberts SG (1999) The role of human TFIIB in transcription start site selection in vitro and in vivo. I Biol Chem 274: 14337-14343.

Hayashi F et al. (1998) Human general transcription factor TFIIB: conformational variability and interaction with VP16 activation domain. Biochemistry 37: 7941-7951.
Kostrewa D et al. (2009) RNA polymerase II-TFIIB structure and mechanism of transcription initiation. Nature 462: 323-330.

Laemmli UK (1970) Cleavage of structural proteins during the assembly of the head of bacteriophage T4. Nature 227: 680-685.

Lagrange T et al. (1998) New core promoter element in RNA polymerase II-dependent transcription: sequence-specific DNA binding by transcription factor IIB. Genes Develop 12: 34-44.

Lakowicz JR (2006) Principles of Fluorescence Spectroscopy. Springer, New York.

Lee S, Hahn S (1995) Model for binding of transcription factor TFIIB to the TBP-DNA complex. Nature 376: 609-612.

Malik S, Lee DK, Roeder RG (1993) Potential RNA polymerase IIinduced interactions of transcription factor TFIIB. Mol Cell Biol 13: 6253-6259.

Nikolov DB et al. (1995) Crystal structure of a TFIIB-TBP-TATA-element ternary complex. Nature 377: 119-128.

Reese JC (2003) Basal transcription factors. Curr Opin Genet Ddevel 13: 114-118.

Roberts SG, Green MR (1994) Activator-induced conformational change in general transcription factor TFIIB. Nature 371: 717-720.

Sievers F et al. (2011) Fast, scalable generation of high-quality protein multiple sequence alignments using Clustal Omega. Mol Systems Biol 7: 539 .

Thompson NE et al. (2009) Minimal promoter systems reveal the importance of conserved residues in the B-finger of human transcription factor IIB. I Biol Chem 284: 24754-24766.

Thomsen $\mathrm{J}$ et al. (2001) The basal transcription factors TBP and TFB from the mesophilic archaeon Methanosarcina mazeii: structure and conformational changes upon interaction with stress-gene promoters. J Mol Biol 309: 589_603.

Tran K, Gralla JD (2008) Control of the timing of promoter escape and RNA catalysis by the transcription factor IIb fingertip. I Biol Chem 283: 15665-15671.

Tsai FT, Sigler PB (2000) Structural basis of preinitiation complex assembly on human pol II promoters. EMBO J 19: 25-36.

Wu WH, Hampsey M (1999) An activation-specific role for transcription factor TFIIB in vivo. Proc Natl Acad Sci USA 96: 2764-2769.

Zhang DY et al. (2000) Intramolecular interaction of yeast TFIIB in transcription control. Nucleic Acids Res 28: 1913-1920.

Zheng L et al. (2004) FRET evidence for a conformational change in TFIIB upon TBP-DNA binding. Eur J Biochem/FEBS J 271: 792800 . 